\title{
Design of Pattern Reconfigurable Patch Antenna Array Based on Reflective Phase-Shifter
}

\author{
Yuqiu Shang $\mathbb{D}^{1},{ }^{1}$ Qingsheng Zeng, ${ }^{1}$ Wanzhao Cui, ${ }^{2}$ Xinwei Wang, ${ }^{3}$ and Gengqi Zheng ${ }^{3}$ \\ ${ }^{1}$ School of Electronics Information Engineering, Nanjing University of Aeronautics and Astronautics, Nanjing, China \\ ${ }^{2}$ National Key Laboratory of Science and Technology on Space Microwave, China Academy of Space Technology (Xi'an), Xi'an, \\ China \\ ${ }^{3}$ School of Electronics Engineering, Xi'an University of Post \& Telecommunications, Xi'an, China \\ Correspondence should be addressed to Yuqiu Shang; shangyuqiu@nuaa.edu.cn
}

Received 22 September 2021; Revised 13 January 2022; Accepted 21 January 2022; Published 11 February 2022

Academic Editor: Renato Cicchetti

Copyright (c) 2022 Yuqiu Shang et al. This is an open access article distributed under the Creative Commons Attribution License, which permits unrestricted use, distribution, and reproduction in any medium, provided the original work is properly cited.

\begin{abstract}
A circularly polarized (CP) broadside antenna array with a continuously adjustable beam is proposed and implemented. First, a reflection phase shifter with varactor diodes loaded on two output terminals of a branch directional coupler is realized. By controlling the voltage across both ends of the varactor diodes in the terminal load circuit, the reactance of the load is changed, allowing control of port phase shift. Following that, the microstrip patch antenna array is combined with the reflection phase shifter to achieve the pattern reconstruction for the array. For verification, a prototype of the designed pattern reconfigurable antenna (PRA) is fabricated and measured. The measurement results indicate that the beam direction of the antenna can be continuously adjusted with a change in the voltage across the varactor diodes. The maximum beam direction of the antenna reaches $\pm 21^{\circ}$ when the biggest DC bias voltage across the varactor diode is chosen as $15 \mathrm{~V}$. Besides, the antenna shows good CP performance in different beam directions.
\end{abstract}

\section{Introduction}

In recent years, reconfigurable antennas have been widely studied for they may alter their performance and function drastically without changing their inherent structures. Besides, since this type of antenna enables easier adjustment of frequencies, polarization, and radiation patterns, it allows reconfigurable antennas to be applied to both satellite and wireless communications [1]. Among reconfigurable antennas, pattern reconfigurable antennas (PRA), which are able to change patterns while keeping their inherent structures unchanged, are the most popular ones, since they may provide wider radiation coverage and an increase in transmitting power and wireless channel capacity [2-8].

With the development and popularization of fifthgeneration mobile communication, access points have become one of the hottest research topics in $5 \mathrm{G}$ in recent years [9]. The antenna beams for wireless access networks need to be adjusted as required. The traditional method of adjusting antenna beams is to change the phase of the phase shifter or the phase-shifted feed network at the back end of the antenna elements of an antenna array. As a result, such a method has seriously increased the complexity and volume of the system. As shown in $[8,10-15]$, PIN diodes were employed as key reconfigurable physical devices for flexible control of forward and backward beam switching, and then a feed network had to be introduced for spurring the PIN diodes. These reconstructed patterns that are either discrete or complementary to each other were created by the on-off effect of diodes or by the discontinuous effect of impedance. Up to now, few documents have been conducted on reconfigurable antennas that are able to continuously change beam pointing and patterns.

Today, the application of new materials in the antenna field has helped to improve reconfigurable antenna design and thus enriched relevant theory [16, 17]. Mechanical methods and phase shifting techniques can be employed to realize reconfigurable antenna designs [18-20]. When phase 
shifting techniques are adopted, variations in reflection phase shift may be continuous, and insertion loss is usually caused by reactive components [21].

In this study, a reflective phase shifter is designed along with a quaternary microstrip antenna array so that a pattern reconfigurable antenna with a continuously adjustable beam can be realized. The beam direction is implemented by adjusting and controlling the voltage loaded on the reactance element. The phase difference between the input and output ports can be continuously changed with the continuous variation of the voltage loaded on the reactance element. In comparison with several of the antennas mentioned above, this antenna could change the beam staring continuously. Although the scanning angle is not big enough, the designed antenna could work as an indoor access antenna installed on the roof of a building, which does not need a bigger scanning angle. And the center frequency of this study is $2.6 \mathrm{GHz}$, which is called channel n4l assigned by China Mobile. And, the maximum beam offset is $\pm 21^{\circ}$, which is sufficient in practical applications.

\section{Antenna Design and Principle of Operation}

2.1. Configuration. As can be seen, the 3-D structure and geometric configuration of the reconfigurable antenna are shown in Figure 1. The reconfigurable antenna array is realized by combining four microstrips circularly polarized (CP) patch elements with their feeding networks. Each radiating element is fed through a reflection phase shifter, and four reflection phase shifters are cascaded with a Wilkinson power divider to form the antenna feeding network. The four patches are etched in four substrate F4BM with a thickness of $4.0 \mathrm{~mm}$, a permittivity of 3.5, and a loss tangent of 0.0035 . The feeding network is also etched on an F4BM substrate with a permittivity of 2.65 and a thickness of $1 \mathrm{~mm}$. Two layers of substrates are laminated together, and the patch antenna and the feeding network are etched in different substrates, respectively. By controlling the voltage at both ends of the varactor diode in the reflection phase shifter, the phase can be continuously adjusted so that the feeding phase of each antenna element, and the beam direction can be continuously changed accordingly. It should be noted that, in the design of the network, the widths of the four sides of the $3 \mathrm{~dB}$ impedance transformation branch directional coupler vary greatly, which is caused by the choice of different impedance ratios.

2.2. Basic Theory of the Reflective Phase Shifter. The reflective phase shifter [22], as shown in Figure 2, is composed of a branch directional coupler and two varactors. Here, a $3 \mathrm{~dB}$ impedance transformation branch directional coupler (ITBC) terminates with two identical loads. When power is fed to port 1 , the same reflective loads connected to port 3 and port 4 reflect power to port 2. $Z_{A}, Z_{B 1}$, and $Z_{B 2}$ are characteristic impedance of the four branches of ITBC and decide the maximum phase shift of each phase shifter where the $Z_{A}, Z_{B 1}$, and $Z_{B 2}$ are expressed as (1).

$$
\begin{aligned}
& Z_{B 1}=Z_{0}, \\
& Z_{B 2}=\frac{Z_{0}}{r_{Z}}, \\
& Z_{A}=\frac{Z_{0}}{\sqrt{2 r_{Z}} .}
\end{aligned}
$$

The terminal reflection coefficient may be expressed as [22]

$$
\begin{aligned}
\Gamma_{L} & =\left|\Gamma_{L}\right| e^{j \Phi_{21}}, \\
& =\frac{\left(r_{Z} R_{s} R_{p}-R_{s} Z_{0}-R_{p} Z_{0}\right)+j X_{L}\left(r_{Z} R_{p}-Z_{0}\right)}{\left(r_{Z} R_{s} R_{p}+R_{s} Z_{0}+R_{p} Z_{0}\right)+j X_{L}\left(r_{Z} R_{p}+Z_{0}\right)},
\end{aligned}
$$

where $r_{Z}$ is the impedance ratio of $Z_{B 1}$ to $Z_{B 2}, R_{s}$ is parasitic resistance of the varactor diode. In Figure $2, C_{v}$ is the junction capacitance of varactor diode, while $L_{S}$ is the parasitic inductance of varactor diode. Different varactor diodes have different parasitic parameters. The type of chosen varactor is SMV2020-079LF with $R_{S}$ being $2.5 \Omega$ and $L_{S}$ being $0.7 \mathrm{nH}$. $C_{V}$ changes with the reverse voltage and ranges from 0.35 to $3.2 \mathrm{pF}$. $L_{0}$ in the circuits is used for matching. $X_{L}$ in (2) is represented as follows:

$$
X_{L}=\omega\left(L_{S}+L_{0}\right)-\frac{1}{\omega C_{V}} .
$$

With the change of the voltage at both ends of the varactor diode, the reactance value of the circuit changes too. Accordingly, the reflection coefficient and the phase between port 1 and port 2 vary so that the design of the pattern reconfigurable antenna can be realized.

2.3. Design of the Pattern Reconfigurable Antenna Array. The impedance ratio $r_{Z}$ and the voltage across the varactor diode are the key parameters to determine the maximum relative phase shift between port 1 and port 2 . The relationship between the maximum phase shift and $r_{Z}$ is shown in Figure 3.

The optimal value of $r_{Z}$ can be selected by the curve in Figure 3. When $r_{Z}$ ranges from about 0 to 1.5 , the maximum value of phase shift increases. When $r_{Z}$ is greater than 1.5, the phase shift decreases. Furthermore, when $r_{Z}$ becomes larger, the difference in the microstrip line width between $Z_{B 1}$ and $Z_{B 2}$ will also become larger, which makes it difficult to manufacture the printed circuits. Therefore, it is very important to select the optimal value of $r_{Z}$ in practice. In this study, choosing $r_{Z}=1.1$ leads to the maximum phase shift of $191.7^{\circ}$ which can be obtained with a reversed voltage of $15 \mathrm{~V}$.

The phase difference is controlled not only by $r_{Z}$ but also by the voltage applied across the varactor. As the reversed junction voltage increases, the PN junction becomes thicker, so the junction capacitance becomes smaller. Meanwhile, the phase shift becomes bigger. When the reverse DC bias is set to $0 \mathrm{~V}$, the junction capacitance is the maximum value, and the phase shift still exists. Hence, for the entire circuit, the difference between the maximum phase shift and the 


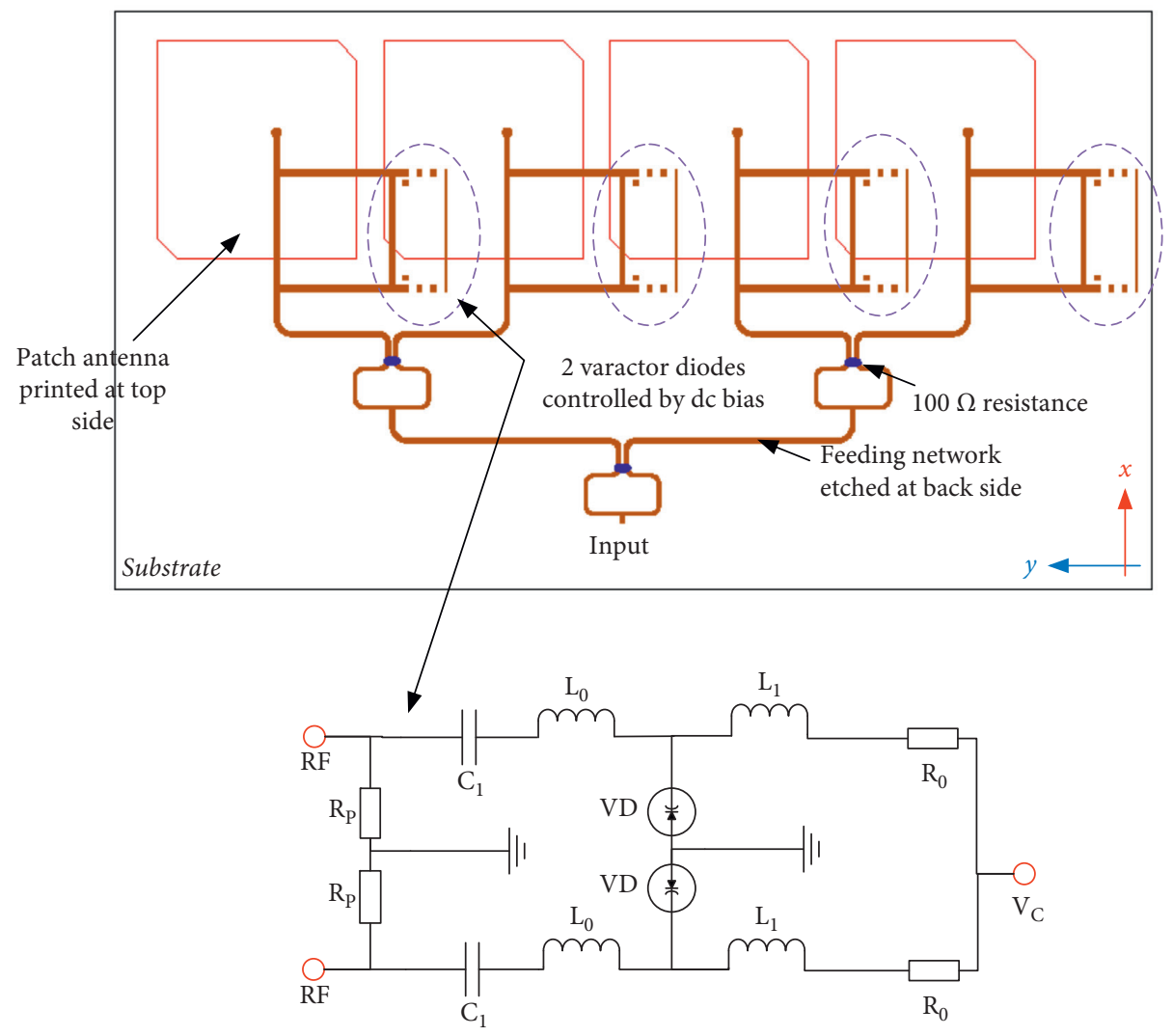

Figure 1: Geometrical configuration of the proposed pattern reconfigurable antenna array.

minimum phase shift is about $190^{\circ}$. Therefore, the maximum difference of the feeding phase between the adjacent antenna elements is $63^{\circ}$. The distance between the elements is $56 \mathrm{~mm}$, which is $0.48 \lambda$ of the center frequency. According to (4), it is found that the maximum direction of the beam points in the direction of $\theta_{M}=68.62^{\circ}$, which biases the direction of vertical to the surface of antenna array by $21.38^{\circ}$.

$$
k d \cos \theta_{M}+\beta=0 \pm 2 \pi m,
$$

where $k=2 \pi / \lambda$ is the wavenumber, $\theta_{M}$ is the maximum direction which biases from the axial of the antenna array, and $\beta$ is the phase difference between adjacent antenna elements. It is assumed here that the leftmost antenna element is the reference element, and its feeding phase is zero degrees. (4) also shows that when the "intraarray phase difference" and "spatial phase difference" between antenna elements are balanced, that is, when the phase provided by the intraarray phase shifter can completely compensate for the phase difference caused by spatial propagation (the two phase values are the same, but the combination is opposite), the maximum value of the antenna pattern is obtained.

\section{Results and Discussion}

3.1. Reconfigurable Antenna Element. A microstrip patch antenna is used for the antenna element, which can radiate circularly polarized waves and has a pair of tangent angles on the patch, as shown in Figure 4. This patch is fed by one probe, which can lead to two radiation modes, $\mathrm{TM}_{01}$ and $\mathrm{TM}_{10}$. The two modes form a circular polarization.

At the same time, the reflection phase shifter is also designed and fabricated, as shown in Figure 5. The values of the components are shown in Table 1.

Figure 6 indicates that the curve of the phase difference between the two ports of the reflection phase shifter varies with the voltage across the varactor diode. It can be seen from Figure 6 that when the applied voltage is greater than $15 \mathrm{~V}$, the rate of phase shift increase slows down, and the maximum phase shift reaches $200^{\circ}$. In this paper, $15 \mathrm{~V}$ is chosen as the highest voltage, and the corresponding phase shift is $190^{\circ}$, while the phase difference between elements has to be chosen as $63^{\circ}$.

In the design of reflective load, capacitor $\mathrm{C}_{1}$ is connected bypass to block DC voltage from being loaded on other varactor diodes through the microstrip line. Inductor $L_{1}$ is connected between DC voltage and a varactor diode, which can prevent RF energy from radiating through the power line. At the designed center frequency of $2.6 \mathrm{GHz}$, the relative phase shift can reach $190^{\circ}$ and change with the variation of voltage. The power dissipation is so small that the electric current is not able to be measured by the meter. The datasheet of the selected varactor diode mentioned that the electric current is only $50 \mathrm{nA}$ under the loaded voltage of $16 \mathrm{~V}$. Its insertion loss changes from 0.8 to $2.1 \mathrm{~dB}$ in the frequency band as shown in Figure 7 .

Figure 8 plots the phase curve for the reflection phase shifter with different loading voltages. Figure 9 illustrates the 


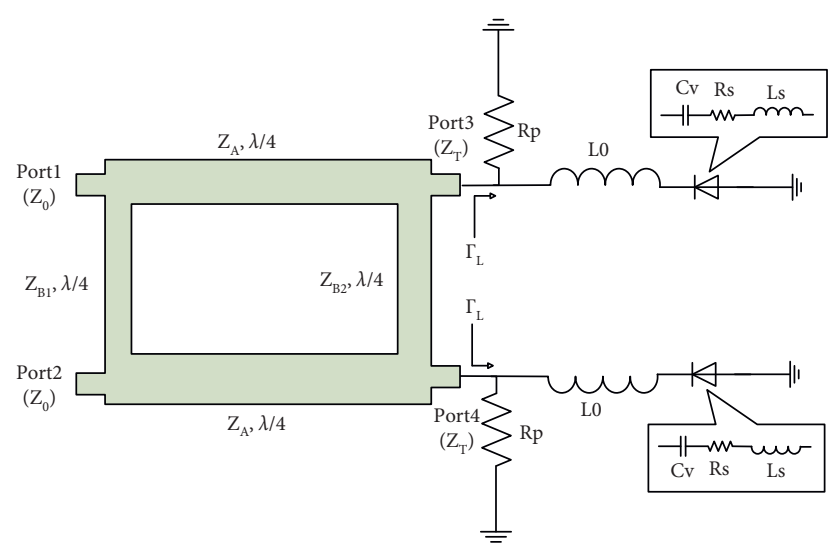

Figure 2: Construction of the reflective phase shifter.

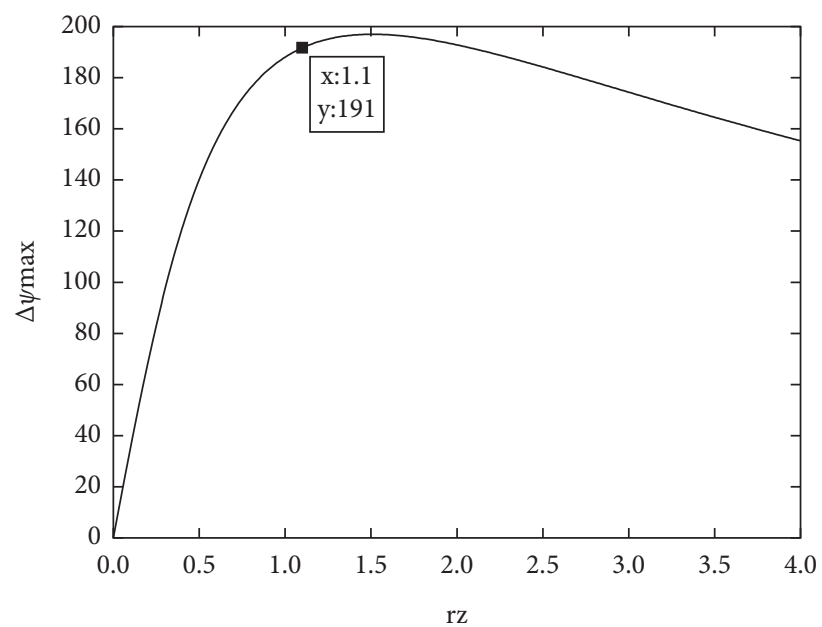

FIgURE 3: The relation curve of phase shift and the $r_{Z}$.

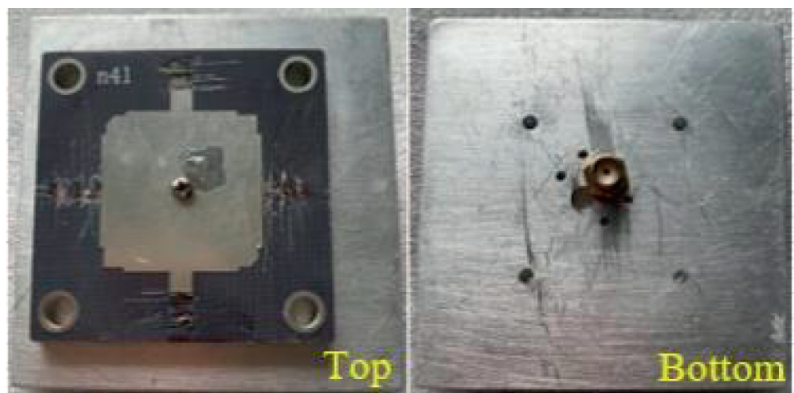

FIgURE 4: Fabrication of microstrip CP patch antenna element.

simulated and measured reflection coefficient and radiation efficiency of the antenna element. It can be seen from Figure 9 that the antenna unit has good matching performances between $2.5 \mathrm{GHz}$ and $2.7 \mathrm{GHz}$, and its radiation efficiency exceeds $80 \%$ in this interval. Figure 10 shows the axial ratio pattern of the antenna element at $2.6 \mathrm{GHz}$.

3.2. Reconfigurable Antenna Array. By cascading four reconfigurable units with a Wilkinson power divider of $1: 4$, a four-element pattern reconfigurable linear array can be obtained, and the fabricated prototype is shown in Figure 11. The dimension size of the entire antenna array is $255 \times 140 \times 5(\mathrm{~mm})^{3}$; meanwhile, the size of the antenna element is $30 \times 30 \times 4(\mathrm{~mm})^{3}$. The measured insertion loss of the feeding network is about $1.9-2.1 \mathrm{~dB}$ in the entire frequency band.

Figure 12 shows the measured and simulated reflection coefficient and radiation efficiency of the proposed antenna. The overlapping impedance bandwidth of the antenna under 


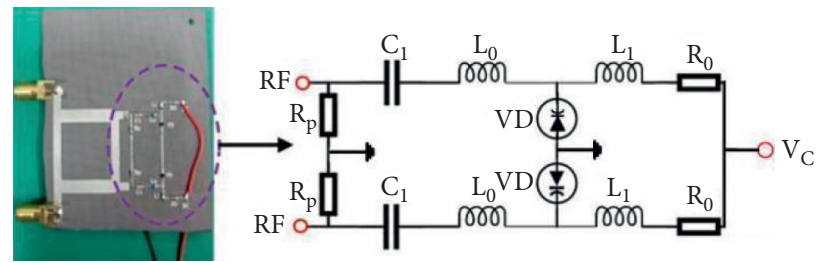

FIGURE 5: Fabrication of reflection phase shifter.

TABLE 1: The values of the components.

\begin{tabular}{ccccc}
\hline $\mathrm{C}_{1}(\mathrm{pF})$ & $L_{1}(\mathrm{nH})$ & $L_{0}(\mathrm{nH})$ & $R_{0}(\Omega)$ & $R_{\mathrm{P}}(\Omega)$ \\
\hline 0.5 & 36 & 2.45 & 1000 & 2000 \\
\hline
\end{tabular}

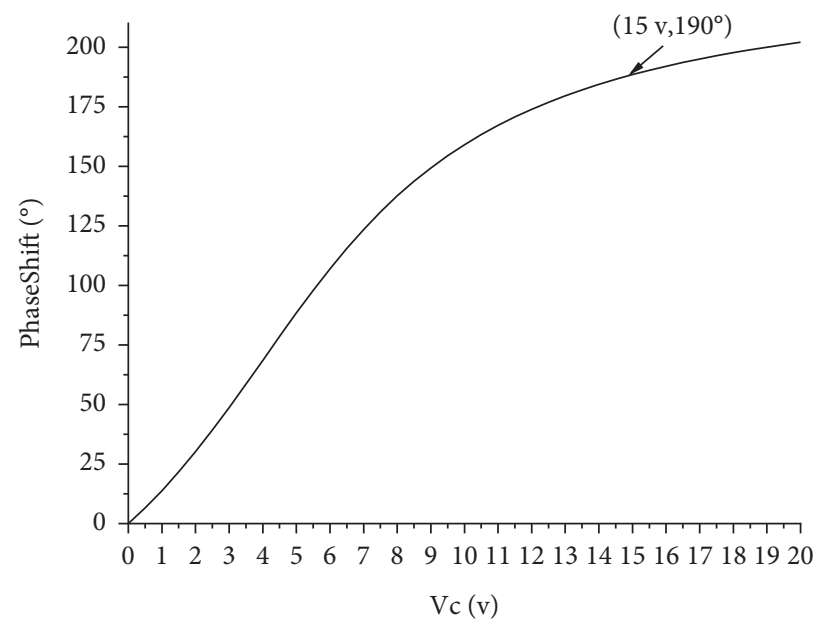

FIgURE 6: Phase shift of reflection phase shifter with loading voltage.

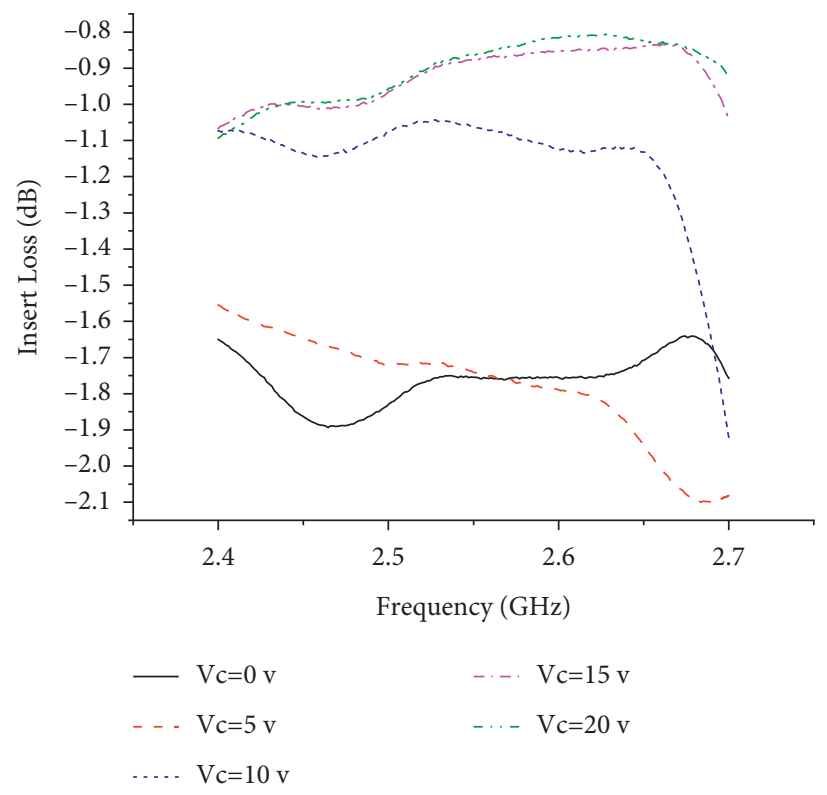

Figure 7: Measured insert loss curve. 


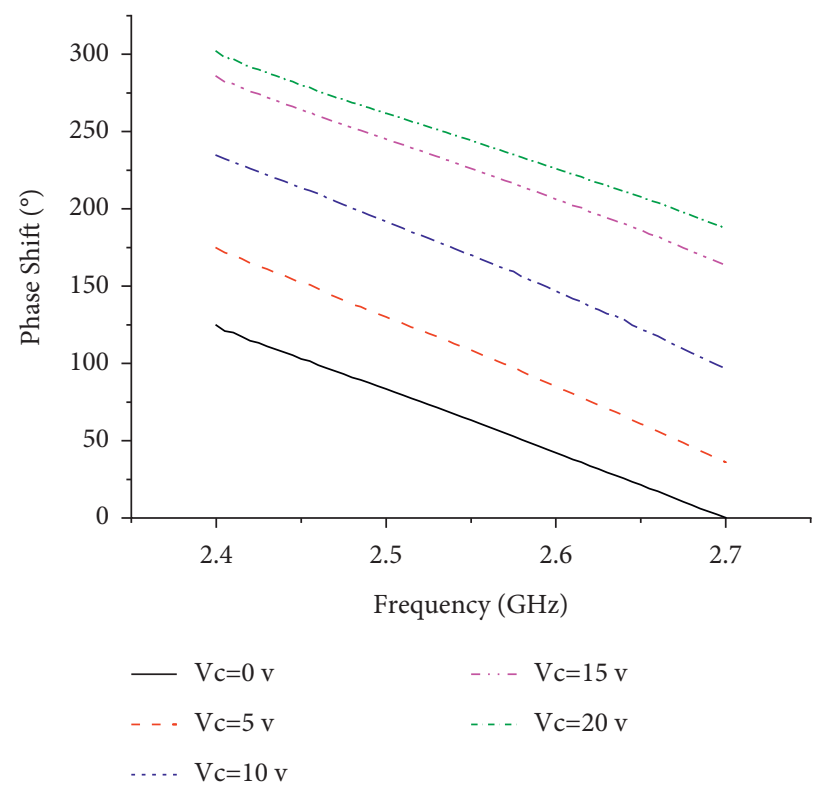

FIGURE 8: The measured phase shift of the reflection phase shifter under different loading voltages.
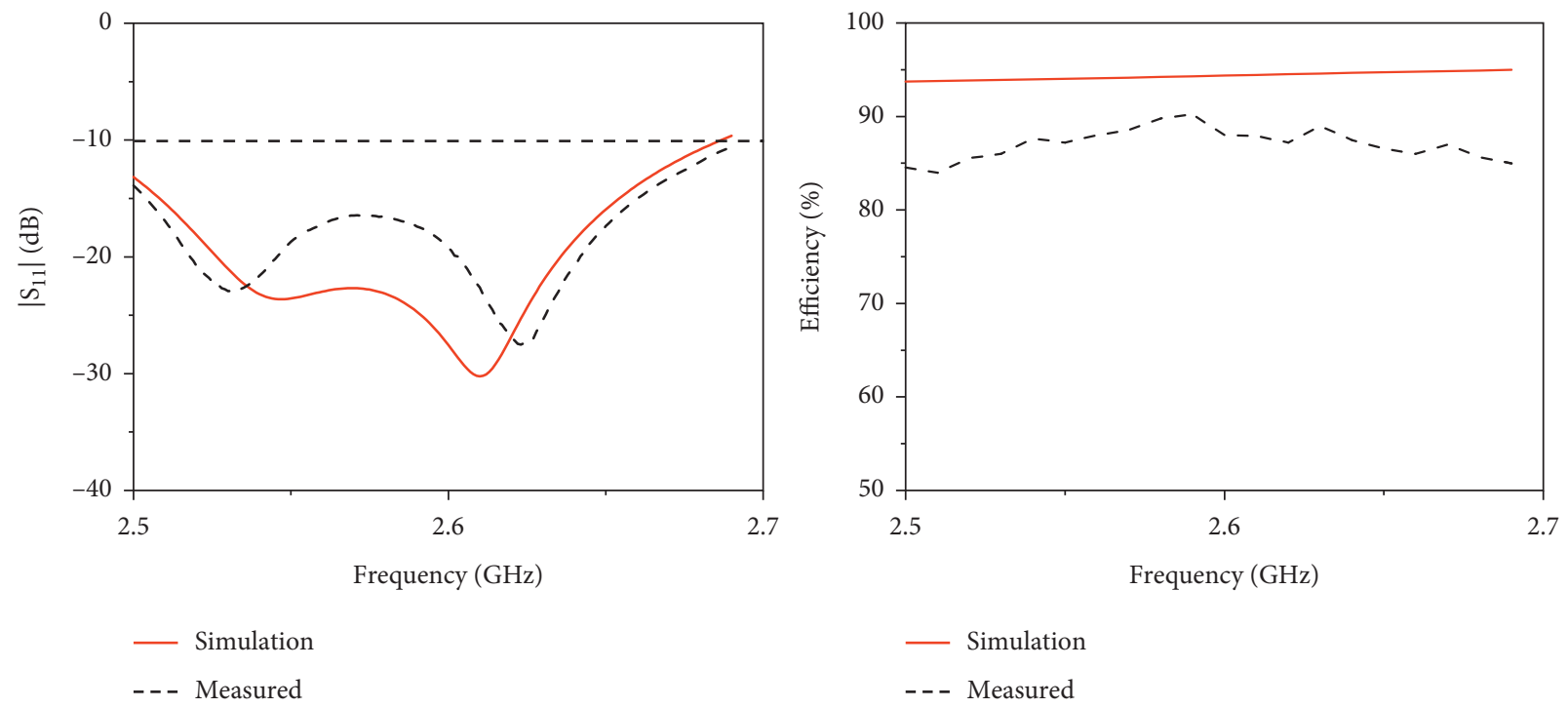

Figure 9: Measured and simulated $\left|S_{11}\right|$ and efficiency of the reconfigurable antenna element.

beam scanning is less than $-10 \mathrm{~dB}$, which is $7.7 \%(2.5-2.7)$. The measured radiation efficiency is nearly $80 \%$ at the central frequency of $2.6 \mathrm{GHz}$ (without scanning) and over $70 \%$ under other scanning conditions.

The maximum beam direction of the antenna is continuously adjusted by the different voltages applied across the varactor diode, as shown in Figure 13. When the phase of the reflection phase shifter is $0^{\circ}, \pm 30^{\circ}$, or $\pm 63^{\circ}$, the maximum gain beams of the array point to five directions: $\pm 0^{\circ}, \pm 10^{\circ}$, or $\pm 21^{\circ}$, respectively. In Figure 13, the bracketed numbers are direction (degree) and gain $(\mathrm{dB})$. Figure 14 shows the measured axial ratio pattern of the antenna array in two orthogonal main planes at $2.6 \mathrm{GHz}$. It can be seen that the antenna has excellent axial ratio characteristics at a $2.6 \mathrm{GHz}$ frequency.

Table 2 provides a comparison between the proposed work and some previously reported PRA, which shows that the proposed antenna has achieved continuous variation of the direction in comparison with the other works. Moreover, $\mathrm{CP}$ is achieved for all operating directions. 


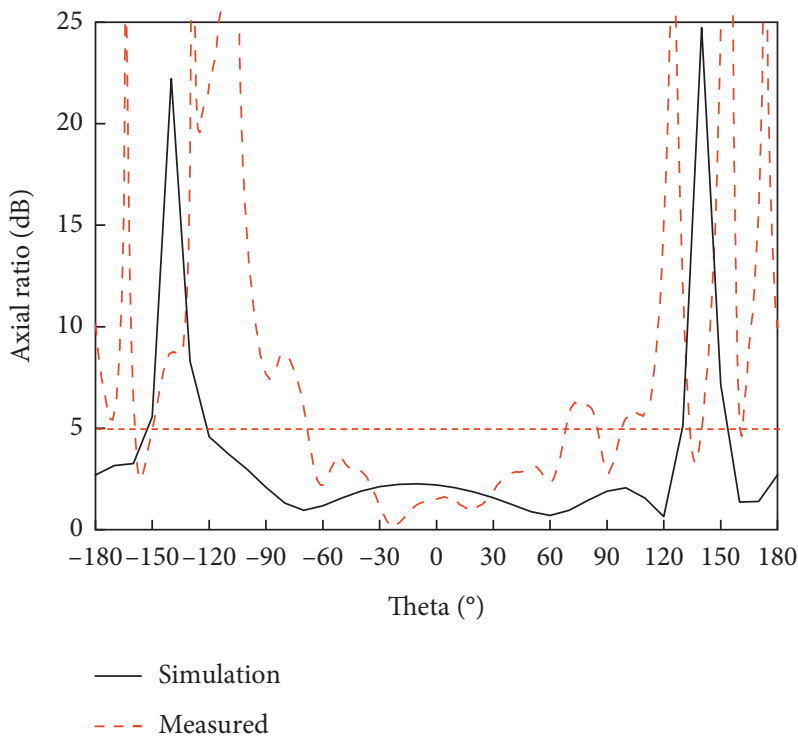

Figure 10: Measured and simulated results of axial ratio at $\phi=0^{\circ}$ for the reconfigurable antenna element (at $2.6 \mathrm{GHz}$ ).

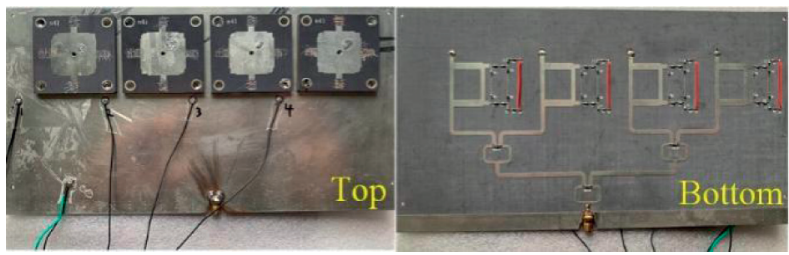

Figure 11: Photograph of the fabricated prototype.
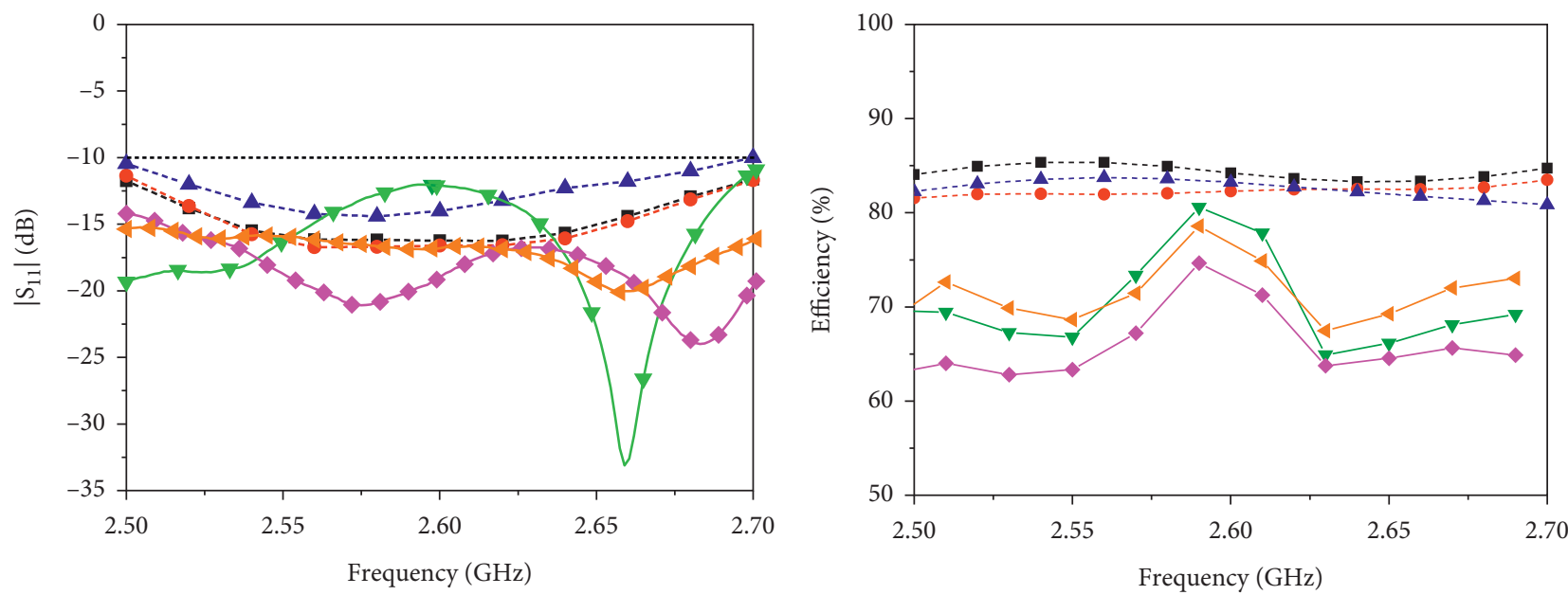

$$
\begin{aligned}
& \text {--m- Sim.phaes shift }\left(0^{\circ}\right) \quad \checkmark \text { Mea.phase shift }\left(0^{\circ}\right) \\
& \text {--๑- Sim.phaes shift }\left( \pm 30^{\circ}\right) \longrightarrow \longrightarrow \text { Mea.phase shift }\left( \pm 30^{\circ}\right) \\
& \text {-- }- \text { - Sim.phaes shift }\left( \pm 60^{\circ}\right) \longleftarrow \text { Mea.phase shift }\left( \pm 60^{\circ}\right)
\end{aligned}
$$

(a)

$$
\begin{aligned}
& \text {--- Sim.Phase Shift }\left(0^{\circ}\right) \quad \rightarrow \text { Mea.Phase Shift }\left(0^{\circ}\right) \\
& \text { - } \bullet \text { Sim.Phase Shift }\left( \pm 30^{\circ}\right) \rightarrow \text { Mea.Phase Shift }\left( \pm 30^{\circ}\right) \\
& \text {-- }- \text { - Sim.Phase Shift }\left( \pm 60^{\circ}\right)-\longleftarrow \text { Mea.Phase Shift }\left( \pm 60^{\circ}\right)
\end{aligned}
$$

(b)

FiguRE 12: Measured and simulated results of the fabricated antenna array: (a) reflection coefficient $\left|\mathrm{S}_{11}\right|$; (b) radiation efficiencies. 


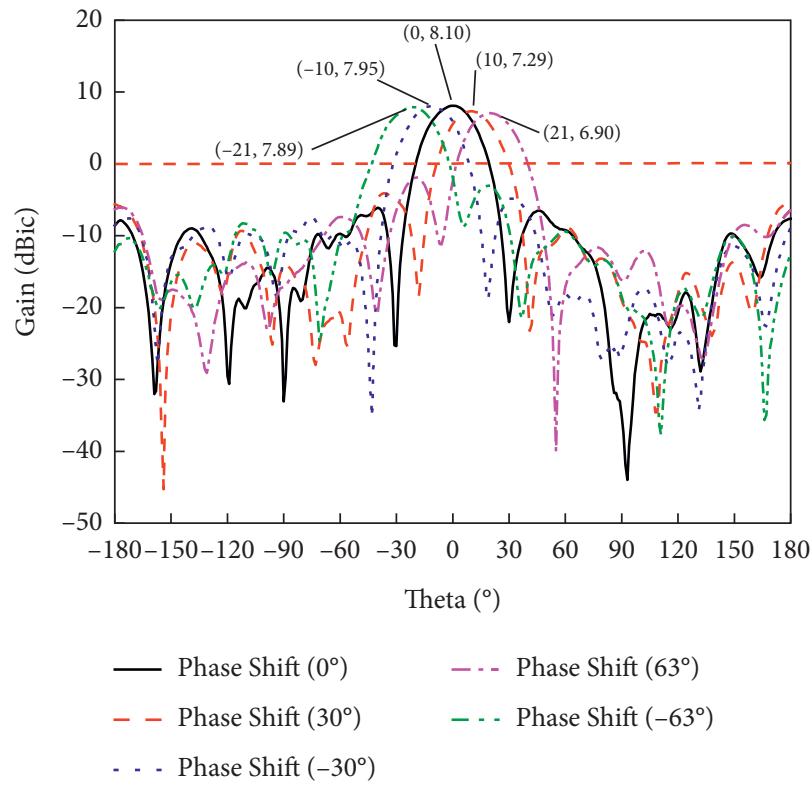

FIgURE 13: Measured normalized radiation patterns of the pattern reconfigurable antenna array at $2.6 \mathrm{GHz}$ on the yoz-plane.

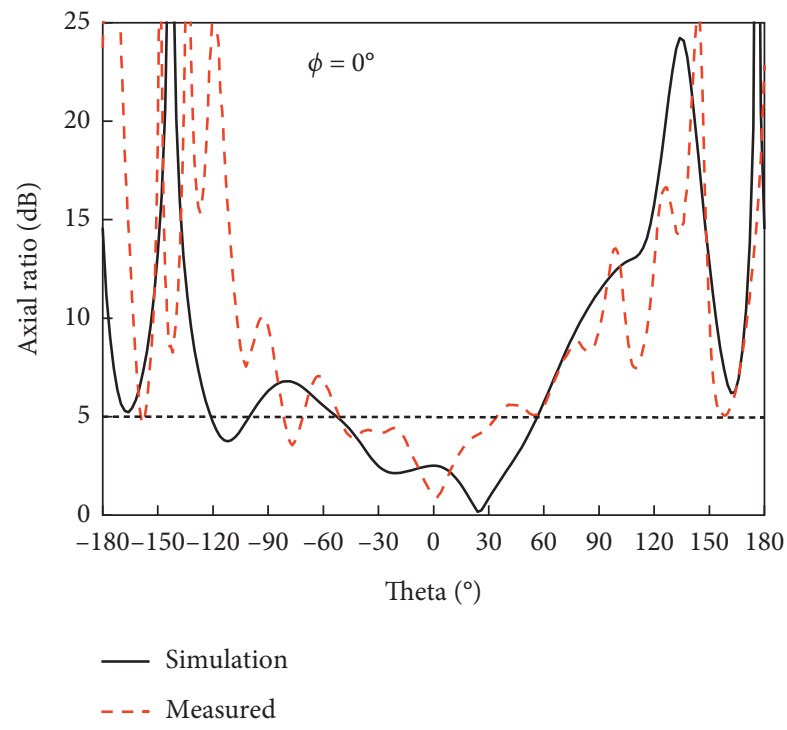

(a)

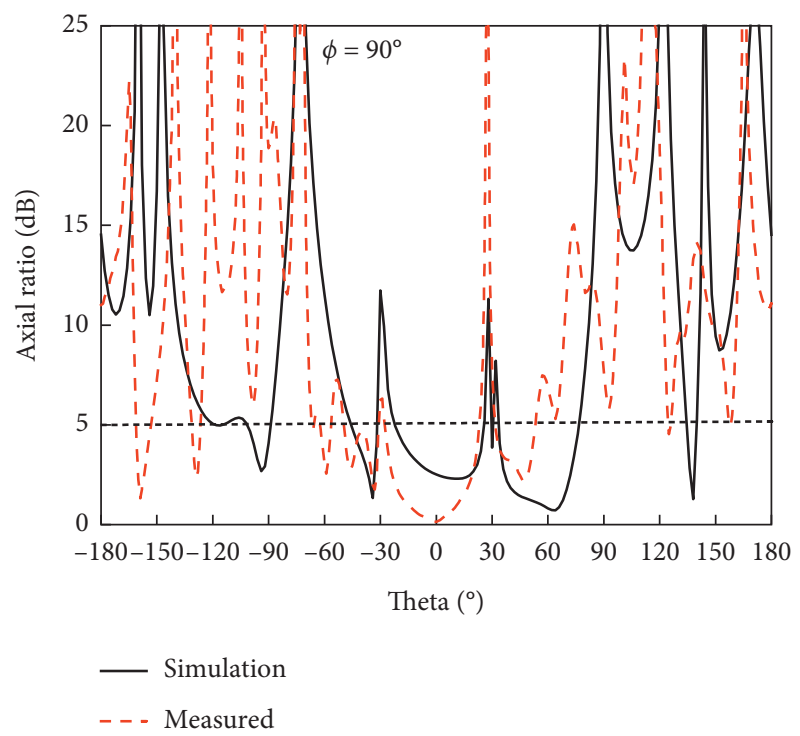

(b)

Figure 14: Measured and simulated axial ratio of proposed antenna array at 2.6 GHz: (a) xoz-plane; (b) yoz-plane.

TABle 2: Comparison between proposed and other reported PRA.

\begin{tabular}{|c|c|c|c|c|c|c|}
\hline Ref. & Ant. type & Pol. & Direction change & Beamwidth $\left({ }^{\circ}\right)$ & Peak gain $(\mathrm{dBi})$ & No. of diodes \\
\hline [2] & Planar monopole & $\mathrm{CP}$ & 4 status & $\mathrm{N} / \mathrm{A}$ & $1.02 / 0.85$ & 8 \\
\hline [4] & End-fired & LP & 3 status & $120^{\circ}$ & 5.86 & 4 \\
\hline [5] & Slot ant. & LP & 4 status & $80^{\circ}$ & $7.0-9.3$ & 14 \\
\hline [6] & Microstrip ant. & LP & 11 status & $60^{\circ}$ & $1.8-2.9$ & 4 \\
\hline$[11]$ & Folded dipoles & LP & 2 status & $150^{\circ} / 75^{\circ}$ & 5 & 4 \\
\hline Prop. & Microstrip ant. & $\mathrm{CP}$ & Continuous variation & $30^{\circ}$ & $6.9-8.1$ & 8 \\
\hline \multicolumn{7}{|c|}{ Ref.: reference; Pol.: polarization; and Prop.: proposed antenna } \\
\hline
\end{tabular}




\section{Conclusion}

This paper proposes a new type of reconfigurable antenna with a continuously adjustable beam pattern that is composed of a reflection phase shifter and a microstrip patch antenna array. To begin with, the phase variation of the two ports of the reflection phase shifter is carefully analyzed, the results of which show that the reactance can be changed by adjusting the voltage loaded on the varactor diode. As a result, the reflection phase of the two ports can be changed accordingly. It is right based on such an idea, a pattern reconfigurable antenna is designed by combining the reflection phase shifter with the microstrip patch antenna array. Measurements show that, with the change of the voltage applied to the two ports of the varactor diode, the beam direction of the antenna can be continuously adjusted, thus realizing the reconfiguration of a pattern. Then, the prototype of the proposed antenna is fabricated, assembled, and tested. Results indicate that when the diode is loaded with five different voltages, the antenna beam can achieve five different directions. Therefore, it can be concluded that the antenna has good pattern reconfiguration performances and good CP performances in different beam directions.

\section{Data Availability}

No data were used to support this study.

\section{Conflicts of Interest}

The authors declare that they have no conflicts of interest.

\section{References}

[1] C. G. Christodoulou, Y. Tawk, S. A. Lane, and S. R. Erwin, "Reconfigurable antennas for wireless and space applications," Proceedings of the IEEE, vol. 100, no. 7, pp. 2250-2261, 2012.

[2] A. Bhattacharjee and S. Dwari, "A monopole antenna with reconfigurable circular polarization and pattern tilting ability in two switchable wide frequency bands," IEEE Antennas and Wireless Propagation Letters, vol. 20, p. 1, 2021.

[3] J. Ren, X. Yang, J. Yin, and Y. Yin, "A novel antenna with reconfigurable patterns using $\mathrm{H}$-shaped structures," IEEE Antennas and Wireless Propagation Letters, vol. 14, pp. 915918, 2015.

[4] Y.-H. Ke, L.-L. Yang, and J. Chen, "A pattern-reconfigurable dielectric resonator antenna based on switchable directors," IEEE Antennas and Wireless Propagation Letters, p. 1, 2021.

[5] H. A. Majid, M. K. A. Rahim, M. R. Hamid, and M. F. Ismail, "Frequency and pattern reconfigurable slot antenna," IEEE Transactions on Antennas and Propagation, vol. 62, no. 10, pp. 5339-5343, 2014.

[6] P. Mahouti, "Design optimization of a pattern reconfigurable microstrip antenna using differential evolution and 3D em simulation-based neural network model," International Journal of RF and Microwave Computer-Aided Engineering, vol. 29, no. 8, Article ID e21796, 2019.

[7] J.-S. Row and C.-W. Tsai, "Pattern reconfigurable antenna array with circular polarization," IEEE Transactions on Antennas and Propagation, vol. 64, no. 4, pp. 1525-1530, 2016.
[8] W. Lin, H. Wong, and R. W. Ziolkowski, "Wideband patternreconfigurable antenna with switchable broadside and conical beams," IEEE Antennas and Wireless Propagation Letters, vol. 16, pp. 2638-2641, 2017.

[9] W. Wonbin Hong, K. Kwang-Hyun Baek, Y. Youngju Lee, Y. Yoongeon Kim, and S. Seung-Tae Ko, "Study and prototyping of practically large-scale mmWave antenna systems for 5G cellular devices," IEEE Communications Magazine, vol. 52, no. 9, pp. 63-69, 2014.

[10] X. Ding and B.-Z. Wang, "A novel wideband antenna with reconfigurable broadside and endfire patterns," IEEE $A n$ tennas and Wireless Propagation Letters, vol. 12, pp. 995-998, 2013.

[11] R. Li, H. Yang, B. Liu, Y. Qin, and Y. Cui, "Theory and realization of a pattern-reconfigurable antenna based on two dipoles," IEEE Antennas and Wireless Propagation Letters, vol. 17, no. 7, pp. 1291-1295, 2018.

[12] R. Wang, B.-Z. Wang, G.-F. Gao, X. Ding, and Z.-P. Wang, "Low-profile pattern-reconfigurable vertically polarized endfire antenna with magnetic-current radiators," IEEE Antennas and Wireless Propagation Letters, vol. 17, no. 5, pp. 829-832, 2018.

[13] T. C. Albuquerque, T. L. V. N. Silva, A. J. R. Serres et al., "Implementation of beam-steering front-ends at $2.45 \mathrm{GHz}$ using modified Wilkinson power dividers, reflection type phase shifters and c-shape monopole antennas: first results," in Proceedings of the 2015 SBMO/IEEE MTT-S International Microwave and Optoelectronics Conference (IMOC), Brazil, November 2015.

[14] J.-C. Chiao, S.-y. Cheng, J. J. L. Chang, I. M. Chio, Y. Kang, and J. Hayasaka, "Mems reconfigurable antennas," International Journal of RF and Microwave Computer-Aided Engineering, vol. 11, no. 5, pp. 301-309, 2001.

[15] Y. Li, M. F. Iskander, Z. Zhang, and Z. Feng, "A new low cost leaky wave coplanar waveguide continuous transverse stub antenna array using metamaterial-based phase shifters for beam steering," IEEE Transactions on Antennas and Propagation, vol. 61, no. 7, pp. 3511-3518, 2013.

[16] S. Ma, G. Yang, F. Meng et al., "Electrically tunable array antenna with beam steering from backfire to endfire based on liquid crystal miniaturized phase shifter," in Proceedings of the 2016 IEEE Conference on Electromagnetic Field Computation (CEFC), Miami, FL, USA, November 2016.

[17] R. K. Gupta, S. E. Anderson, and W. J. Getsinger, "Impedancetransforming 3-dB $90^{\circ}$ hybrids," IEEE Transactions on Microwave Theory and Techniques, vol. 35, no. 12, pp. 1303-1307, 1987.

[18] C. Chien-San Lin, S. Sheng-Fuh Chang, and W. Wen-Chun Hsiao, "A full-360\$\{ \circ\}\$ reflection-type phase shifter with constant insertion loss," IEEE Microwave and Wireless Components Letters, vol. 18, no. 2, pp. 106-108, 2008.

[19] A. Ramadan, M. Al-Husseini, Y. Tawk, K. Y. Kabalan, and A. El-Hajj, "A novel frequency/pattern-reconfigurable microstrip antenna for WLAN applications," in Proceedings of the Fourth European Conference on Antennas and Propagation, Barcelona, Spain, April 2010.

[20] S. Shamsinejad, N. Khalid, F. M. Monavar et al., "Pattern reconfigurable cubic slot antenna," IEEE Access, vol. 7, pp. 64401-64410, 2019.

[21] S. Zhang, G. H. Huff, J. Feng, and J. T. Bernhard, "A pattern reconfigurable microstrip parasitic array," IEEE Transactions on Antennas and Propagation, vol. 52, no. 10, pp. 2773-2776, 2004. 
[22] C. Lin, S. Chang, C. Chang, and Y. Shu, "Design of a reflection-type phase shifter with wide relative phase shift and constant insertion loss," IEEE Transactions on Microwave Theory and Techniques, vol. 55, no. 9, pp. 1862-1868, 2007. 